METABOLISM

\section{Chill out}

Many mammals actively reduce their body temperature to go into energy-conserving states. However, the neural mechanisms the mediate these states are unknown. Now, Hrvatin et al. and Takahashi et al. identify populations of hypothalamic neurons in rodents that drive torpor and a hibernation-like state, respectively.

Although mice do not hibernate, when fasted, they do show daily torpor: a reduction in body temperature and locomotion for up to 24 hours. Hrvatin et al. used targeted recombination in active populations (TRAP) to express a designer receptor exclusively activated by a designer drug (DREADD) in neurons active as mice went into fasting-induced torpor. Subsequent activation of these torpor-TRAPed neurons induced torpor without fasting.

To determine the location or locations containing these torporassociated neurons, Hrvatin et al. used viral injections to target the
TRAP construct to different parts of the brain. Only when the anterior and ventral parts of the medial and lateral preoptic area (avMLPA) were transduced did chemogenetic activation of torpor-TRAPed neurons induce torpor-like state, implying that torpor-associated neurons are restricted to this area.

Using single-nucleus RNA sequencing, Hrvatin et al. found that about $43 \%$ of torpor-TRAPed neurons were glutamatergic neurons expressing the gene encoding adenylate cyclase-activating polypeptide 1 (Adcyap1). In line with this, chemogenetic activation of avMLPA neurons expressing the glutamate neuron marker Vglut2 or Adcyap 1 induced key features of torpor. Moreover, silencing of avMLPA $^{\text {Vllut } 2}$ or avMLPA Adcyap 1 neurons (using tetanus toxin) disrupted fasting-induced torpor. $\operatorname{avMLPA}^{\text {Adcyap } 1}$ neurons are a subset of avMLPA ${ }^{\text {glut2 }}$ neurons, and calcium imaging of avMLPA ${ }^{\text {Adcyap } 1}$ neurons revealed that these neurons show a unique pattern of activity with the onset of torpor and maintain this activity until exiting torpor. Together, these results suggest that avMLPA ${ }^{\text {Adcyap } 1}$ neurons are needed for torpor.

In the other study, Takahashi et al. investigated neurons expressing pyroglutamylated RFamide peptide (QFRP), which has been implicated in food intake and sympathetic regulation. Chemogenetic activation of QFRP-expressing neurons in the anteroventral periventricular nucleus, medial preoptic area and periventricular nucleus (which the authors collectively called 'Q' neurons) induced reductions of skin temperature, locomotion and metabolism lasting more than 48 hours.

Q neurons project strongly to the dorsomedial hypothalamus (DMH). Optogenetic activation of $\mathrm{Q}$ neuron bodies, or $\mathrm{Q}$ neuron terminals in the DMH induced robust hypothermia, whereas optogenetic deactivation of DMH terminals of Q neurons stopped the hypothermia driven by excitation of these same terminals.

Takahashi et al. analysed the behaviour, body temperature and oxygen consumption of mice undergoing Q-neuron-induced hypothermia (QIH) at different ambient temperatures. From these measures, they inferred that, during QIH, the animal's heat conductance does not reduce as it does during torpor, but that instead the animal's set-point temperature is lowered, as occurs in hibernation. In line with this, the animals' posture during QIH at an ambient temperature of $28^{\circ} \mathrm{C}$ was extended (as typically seen of animals in a hot environment), whereas animals undergoing fasting-induced torpor at $28^{\circ} \mathrm{C}$ typically show a sitting behaviour associated with energy conservation. Moreover, metabolism was increased during QIH at low ambient temperature $\left(16^{\circ} \mathrm{C}\right)$ compared with during $\mathrm{QIH}$ at $20^{\circ} \mathrm{C}$ or $28^{\circ} \mathrm{C}$, indicating a metabolic similarity to hibernation. Thus, QIH is different to daily torpor but similar to hibernation.

Co-labelling of Q neurons revealed that some of these cells are excitatory (expressing Vglut2), some are inhibitory (expressing $V g a t$ ) and some are 'hybrid' neurons expressing both Vgat and Vglut2. Knocking out Vgat or Vglut2 from Q neurons disrupted the QIH induced by chemogenetically activating these neurons, suggesting that both glutamatergic and GABAergic transmission are needed for QIH.

Together, these studies shed light on the circuit bases of torpor and a hibernation-like state not typically seen in mice.

Natasha Bray

ORIGINAL ARTICLES Hrvatin, S. et al. Neurons that regulate torpor. Nature https://doi.org/10.1038/ 541586-020-2387-5 (2020) | Takahashi, T. M. et al. A discrete neuronal circuit induces a hibernationlike state in rodents. Nature https://doi.org/10.1038/ s41586-020-2163-6 (2020) 